\title{
Gastric Partitioning Bypass Procedure: A Novel Technique in Bariatric Surgery
}

\section{Afsharfard $\mathrm{A}^{1}$, Gholizadeh $\mathrm{B}^{\star_{2}}$ and Azimi $\mathrm{B}^{1}$}

\author{
${ }^{1}$ Shohadaye Tajrish Medical Center, Shahid Beheshti University of Medical Sciences, Tehran, Iran \\ ${ }^{2}$ Modarres Medical Center, Shahid Beheshti University of Medical Sciences, Tehran, Iran
}

${ }^{*}$ Corresponding author: Gholizadeh B, MD, Assistant Professor of General Surgery, Modarres Medical Center, Shahid Beheshti University of Medical Sciences, Tehran, Iran, 1998734383, Tel: +98-914-382-1369, E-mail: barmak.gholizadeh@Gmail.com
Citation: Afsharfard A, Gholizadeh B, Azimi B (2018) Gastric Partitioning Bypass Procedure: A Novel Technique in Bariatric Surgery. J Surg Oper Care 3(1): 101. doi: 10.15744/2455-7617.3.101

Received Date: September 29, 2017 Accepted Date: October 25, 2017 Published Date: October 27, 2017

\begin{abstract}
Objective: The current standard of care offers surgery as the most favored treatment modality for morbidly obese patients to achieve weight loss. The currently available surgical techniques have some limitations and introduction of novel techniques is inevitable. The aim of this study is to introduce the novel gastric partitioning bypass technique and assess its effectiveness and adverse effects.

Methodology: Thirty individuals were randomly recruited from a population who were eligible for bariatric surgery. The subjects had a baseline visit and were followed-up after surgery assessing weight loss and adverse effects related to the surgery.

Results: The study population included 30 patients (Mean age $41 \pm 5$ and $93 \%$ female). Mean BMI and excess body weight at the baseline were $49 \pm 7 \mathrm{~kg} / \mathrm{m}^{2}$ and $79 \pm 15 \mathrm{Kg}$, respectively. Median follow-up time was 36 months and 27 (90\%) patients completed the study. Excess weight loss was $54.76 \pm 13.76 \%$ and $60.43 \pm 14.49 \%$ after 12 and 48 months, respectively. In post-operative period Two patients (6.6\%) developed surgical site infection, other complications such as bleeding or anastomotic leakage, or any complication requiring emergent surgery were not detected.
\end{abstract}

Conclusion: The novel gastric partitioning bypass technique can serve as an effective and reliable technique in bariatric surgery.

Keywords: Morbid obesity; Bariatric surgery; Gastric bypass

\section{Introduction}

Morbid obesity as a chronic condition remains one of the major health problems of the present generation. In 2012, over onethird of US adults were identified as obese and the prevalence has been growing since, such that currently, obesity is considered as an epidemic [1]. In addition to developed countries, obesity has recently become a health concern in developing nations as well. For example, a recent study reported that the total prevalence of obesity in 1999-2001 was $15.8 \%$ and 31.3\% in Iranian men and women, respectively. The prevalence has increased to $21.1 \%$ in men and $38.6 \%$ in women in 2006 through 2008 [2,3]. Obesityrelated comorbidities include a wide range of conditions such as hypertension, diabetes, coronary artery disease, dyslipidemia, hypertriglyceridemia, obstructive sleep apnea, osteoarthritis, social isolation, and depression [4]. Studies have shown that obesity is associated with increased risk of various neoplasms such as breast, colorectal, esophageal, hepatobiliary and prostate cancer [1,5]. While lifestyle modifications and exercise have been shown to be effective as first line intervention in obese individuals, these modalities have little effect on morbidly obese patients and bariatric surgery remains the most efficient treatment option in this patient population [6,7].

There are three main surgical techniques in bariatric surgery: restrictive, malabsorptive, and combined restrictive/malabsorptive [8]. Commonly used surgical techniques are vertical banded gastroplasty (VBG), laparoscopic adjustable gastric banding (LAGB), laparoscopic Roux-en-Y gastric bypass (LRYGB), and sleeve gastrectomy. All of these methods are shown to be effective; however, they are not without complications. VBG and LAGB use mesh or silastic band [9-11] which remain in the abdomen and are associated with foreign body related intra-abdominal infections or intestinal obstruction requiring emergent laparotomy in 33.1 to 48 percent of patients [12-16]. Purely restrictive techniques such as sleeve gastrectomy do not result in persistent weight loss and thus sometimes require revision surgeries due to secondary weight gain. For example, incidence of revision surgery in sleeve gastrectomy is about $20 \%$ [17] and in LAGB is 8.3 to $18.1 \%$ [15,16,18]. LRYGB technique is a restrictive/malabsorptive technique leading to significant and persistent weight loss. The only caveat is that since the pouch is completely detached from remaining part of stomach, potential esophagogastroduodenoscopy (EGD) will be a challenge in the future [5,19]. In addition, LRYGB is 
known to be associated with nutritional deficiencies such as anemia and bone disease secondary to bypass of the alimentary tract with absorptive capacity [5,19]. Also, LRYGB and LAGB techniques are advanced laparoscopic techniques requiring highly trained surgeons and expensive equipment.

An ideal surgical technique must not only be effective in producing significant and durable weight loss, but also must be safe, leading to few side-effects and metabolic sequelae. Moreover, the surgical technique needs to be simple with reasonable learning curve for trainees, and the required equipment needs to be widely accessible in regular operating rooms. The purpose of the current study is to introduce the new method of "open gastric partitioning bypass" and assess the efficacy and side effect profile of this new method. This technique utilizes both restrictive and malabsorptive techniques for weight reduction and prevention of secondary weight gain. In contrast to VBG and gastric banding, this method does not leave a foreign body in the abdomen. This is a relatively simple procedure without requirement for advanced or expensive equipment in contrast to LRYGBP and LAGB. Furthermore, in contrast to LRYGBP, should complications occur in upper GI structures, EGD is feasible after this procedure.

\section{Methods}

We prospectively recruited 30 individuals over the course of 4 years from April 2012 to April 2016 who were eligible for bariatric surgery. The inclusion criteria for enrolment were body mass index (BMI) of $40 \mathrm{~kg} / \mathrm{m}^{2}$ or higher; or BMI between 35 and $40 \mathrm{~kg} /$ $\mathrm{m}^{2}$ with obesity-related comorbidity as described in previous studies [20,21]. The subjects provided written informed consent to undergo open gastric partitioning bypass procedure and the study protocol was approved by the Institutional Review Board.

According to the study protocol, the subjects had a baseline visit prior to surgery and were followed-up by post-op clinical visits on 1, 3, 6, 12, 18, 24, 36, and 48 months after the surgery. Each study visit included measuring weight, gathering information about the potential side effects of surgery such as pain at the surgical site, or experiencing other symptoms such as nausea, vomiting, or heartburn. Venous blood was drawn in each visit for assessment of complete blood count and comprehensive metabolic panel. Nutritional supplements were provided if any of the patients had evidence of nutritional deficiencies.

Statistical analyses were performed by SPSS software (version 22.0, SPSS Inc., Chicago, IL). Continuous variables are reported as mean $\pm \mathrm{SD}$. We used student T-test to analyze normally distributed continuous variables and Whitney-Mann test for nonnormally distributed variables. Categorical variables were analyzed by using Chi-Square test. Statistical significance is defined as two-sided p-value $<0.05$.

\section{Gastric Partitioning Bypass Operative Technique}

We developed for the first time a "Gastric Partitioning Bypass" (GPB) technique for bariatric surgery. This surgical method has two main components as demonstrated (Figure 1). The first component is restrictive and involves creation of a gastric pouch with suturing anterior and posterior gastric walls near the lesser curvature similar to the procedure performed in vertical banded gastroplasty. The second component is mal-absorptive and involves bypassing the stomach.

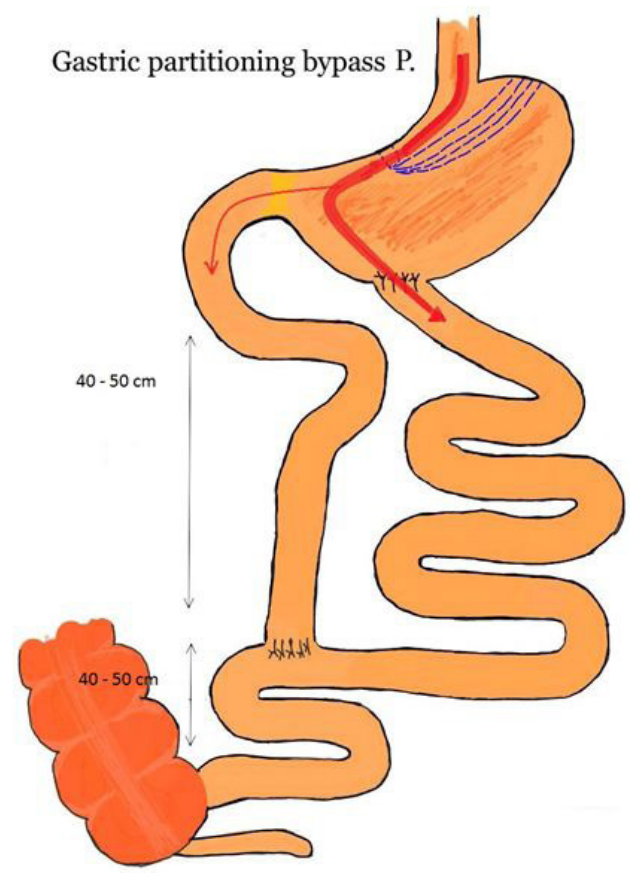

Figure 1: This surgical method has two main components: The first component is restrictive and involves creation of a gastric pouch with suturing anterior and posterior gastric walls near the lesser curvature (dotted lines) similar to the procedure performed in vertical banded gastroplasty. The second component is mal-absorptive and involves bypassing the stomach in roux-en-y technique which creates biliopancreatic limb and common channel with approximate lengths of 40 to $50 \mathrm{~cm}$. Arrows represent passage of stomach contents to these two limbs (roux limb > biliopancreatic limb) 
After a midline epigastric incision, we performed gastrolysis by releasing the gastrocolic omentum from the greater curvature (antrum through cardia). Then, we sutured the anterior and posterior walls of the stomach together in a simple separate mattress style. We used four-row suture line starting $4 \mathrm{~cm}$ distal to cardia in greater curvature up to crow's foot region in the lesser curvature side, so that the distal pouch exit on the lesser curvature side would be about $1.5 \mathrm{~cm}$. Then, we performed a Roux-en-y gastric bypass in a manner that biliopancreatic limb is $50 \mathrm{~cm}$, common channel $50 \mathrm{~cm}$, and for patients with high BMI $\left(>45 \mathrm{~kg} / \mathrm{m}^{2}\right) \operatorname{sizes}$ are determined $40-50 \mathrm{~cm}$. Moreover, if gallstones were present, the gallbladder was removed.

Prior to general anesthesia, we used epidural anesthesia so that in the post-operative period the patient could breathe deeply and expectorate bronchial secretions. Prophylactic antibiotics were administered $2 \mathrm{~h}$ before and for $24 \mathrm{~h}$ after the operation per hospital protocols. Low-dose heparin and intermittent calf compressions were used to prevent deep venous thrombosis. The patients were transferred to the surgical intensive care unit for $24 \mathrm{~h}$ after the operation. We initiated the diet with liquids and gradually advanced as patients tolerated. The average post-op hospital stay was 5 days. In addition to GPB, twelve patients (40\%) underwent elective abdominoplasty for cosmetic indications.

\section{Results}

The study population included 30 patients (Mean age $41 \pm 5$ and $93 \%$ female). Mean BMI and excess body weight at the baseline were $49 \pm 7$ and $79 \pm 15 \mathrm{~kg}$, respectively. Mean operative time was $143 \pm 36$ minutes and mean blood loss was $350 \pm 100 \mathrm{cc}$. Details of baseline characteristics are summarized in (Table 1). Median follow-up time was 36 months and follow-up information was available for 27 (90\%) of study subjects. Twenty-six patients (86.6\%) were followed for 36 months and 24 (80\%) for 48 months. There was no intra- or post-operative mortality during follow-up period. Two patients (6.6\%) developed surgical site infection, which was managed by incision, drainage and a short course of antibiotic therapy. We did not observe other complications such as bleeding, anastomotic leakage, deep venous thrombosis, small bowel obstruction or any complication requiring emergent surgery. There was no evidence of liver or renal dysfunction, electrolyte abnormalities, or anemia (Table 2).

\begin{tabular}{|c|c|c|}
\hline Demographic & Characteristic & Patients $(\mathbf{n}=\mathbf{3 0})$ \\
\hline Gender & Male, \% (n) & $6.7 \%(\mathrm{n}=2)$ \\
\hline & Female, \% (n) & $93.3 \%(\mathrm{n}=27)$ \\
\hline Age (yr.) & Mean (SD) & $41.5(7.32)$ \\
\hline & Range & $(25,63)$ \\
\hline Body weight (kg) & Mean (SD) & $135.2(13.6)$ \\
\hline & Range & $(112,157)$ \\
\hline BMI $\left(\mathrm{kg} / \mathrm{m}^{2}\right)$ & Mean (SD) & $49.94(6.63)$ \\
\hline & Range & $(37.7,62.2)$ \\
\hline EW (kg) & Mean (SD) & $79.5(15.1)$ \\
\hline & Range & $(51.7,102.5)$ \\
\hline Follow up (mo.) & Mean (SD) & $41.2(15.65)$ \\
\hline
\end{tabular}

BMI: Body Mass Index; EW: Excess Weight

Table 1: Baseline demographic characteristics of the study subjects

\begin{tabular}{|c|c|ll|}
\hline Lab. test & Result (SD) & \multicolumn{2}{|c|}{ Normal range } \\
\hline Aspartate aminotransferase & $28.4(10.01)$ & 5 to 40 & international units per liter \\
\hline Alanine transaminase & $23.68(8.81)$ & 7 to 56 & international units per liter \\
\hline Alkaline phosphatase & $197.40(17.5)$ & 44 to 147 & international units per liter \\
\hline Total Bilirubin & $0.34(0.11)$ & 0.3 to 1.9 & milligrams per deciliter \\
\hline Direct bilirubin & $0.14(0.04)$ & 0 to 0.3 & milligrams per deciliter \\
\hline Creatinine & $0.9(0.66)$ & $0.5-1.5$ & milligrams per deciliter \\
\hline Hemoglobin (men) & $14.75(0.35)$ & 13.5 to 17.5 & grams per deciliter \\
\hline Hemoglobin (women) & $12.90(0.68)$ & 12.0 to 15.5 & grams per deciliter \\
\hline Serum Calcium & $9.79(0.66)$ & $8.5-10.2$ & milligrams per deciliter \\
\hline
\end{tabular}

SD: Standard deviation

Table 2: Laboratory test results after 48 months of follow-up in 24 patients

All patients demonstrated significant weight loss postoperatively such that excess weight loss (EWL) after 12-month follow-up was $54.76 \pm 13.76 \%, \mathrm{p}<0.001$. Similarly, EWL was $65.17 \pm 9.16 \%, 62.83 \pm 13.76$, and $60.43 \pm 14.49 \%$ after 24 , 36 , and 48 months, respectively ( $\mathrm{p}<0.001$ for all) (Figure 2). Mean postoperative total weight loss (\%TWL) at 12, 24, 36 and 48 months was $29.9,34.6$, 33.5 , and $32.9 \%$, respectively. The mean body mass index (BMI) significantly decreased from $49.94 \pm 6.63 \mathrm{~kg} / \mathrm{m}^{2}$ to $32.19 \pm 4.98$ $\mathrm{kg} / \mathrm{m}^{2}(\mathrm{p}<0.001)$ representing a $35.54 \%$ reduction in BMI over a 48-month period. In addition, we observed a significant weight 
loss (86.13 \pm 13.6 at baseline vs.135.26 $\pm 13.6 \mathrm{~kg}$ at 48 months post-op, $\mathrm{p}<0.001)$, representing a $36.32 \%$ weight loss over a 48 -month period.

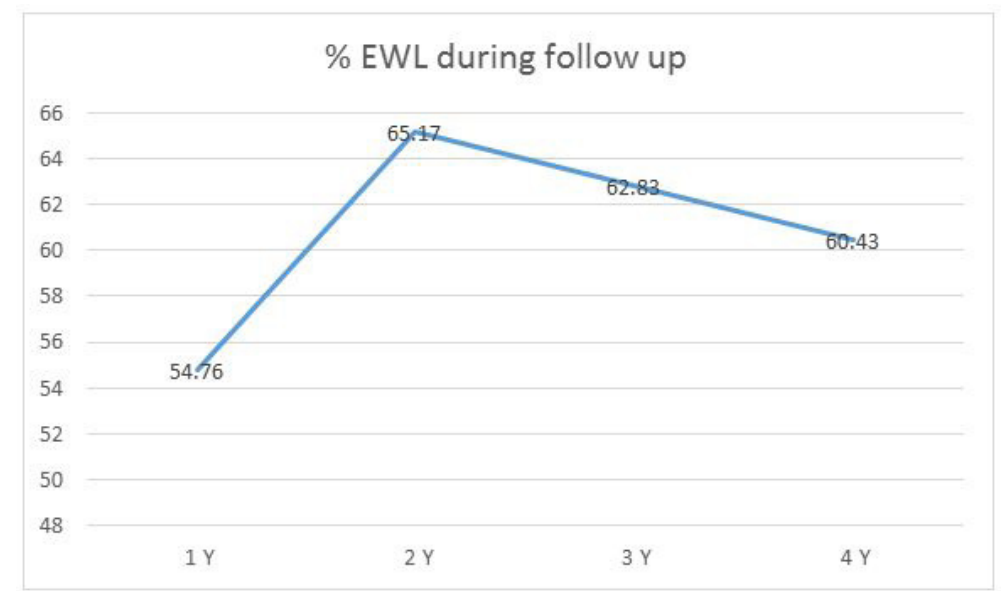

Figure 2: This figure demonstrate changes of \%EWL during 48 month follow up period. Weight loss was achieved in the first 24 months and remains persistent during the follow-up period

The comorbid conditions identified in the study population are summarized in (Table 3). The most common of these comorbidities was musculoskeletal complaints associated with obesity, which significantly improved after the bariatric surgery. It is worth noting that all patients with a preoperative diagnosis of obstructive sleep apnea requiring treatments such as nightly continuous positive airway pressure (CPAP), reported that they no longer required treatment after their weight loss by the 12-month post-operative follow-up visit. Furthermore, five of six patients who were taking antihypertensive medications preoperatively were successfully weaned off of these medications postoperatively after one year. The statistical analysis of preexisting condition improvement was inconclusive, given the small sample size.

\begin{tabular}{|c|c|c|}
\hline Pre-existing medical condition & N (\% of total) & Improvement of condition \\
\hline Diabetes mellitus type 2 & $1(3.3 \%)$ & $1(100 \%)$ \\
\hline Hypertension & $6(24 \%)$ & $5(83 \%)$ \\
\hline Asthma & $4(16 \%)$ & $3(75 \%)$ \\
\hline Hyperlipidemia & $8(32 \%)$ & $5(62.5 \%)$ \\
\hline Obstructive sleep apnea & $9(36 \%)$ & $9(100 \%)$ \\
\hline Musculoskeletal complaints & $20(80 \%)$ & $18(90 \%)$ \\
\hline Stress incontinence & $4(16 \%)$ & $4(100 \%)$ \\
\hline GERD & $10(40 \%)$ & $9(90 \%)$ \\
\hline
\end{tabular}

Table 3: Comorbid conditions and their improvement after weight loss

\section{Discussion}

In this article, we present our trial of a new open gastric partitioning bypass procedure for morbidly obese patients. The results were promising as \%EWL was 65\% after 24 months and 60\% after 48 months, and we observed acceptable post-operative complications - only two surgical site infections and no nutritional deficiencies or emergent operations.

Lower rate of complications or requirement for re-operation in GPB is likely secondary to the unique technique. In contrast to VBG and adjustable gastric banding techniques, no foreign body such as mesh, silastic band, subcutaneous reservoir, or connecting tube is utilized in this technique. Avoiding foreign bodies would reduce the complication rates such as re-operation, which is one of the challenges in adjustable gastric banding procedure (63\% reoperation rate and a 48\% band-removal rate) [12-14].

Similar to other bariatric surgery techniques, we demonstrated that the obesity-related comorbidities are significantly improved or completely resolved with weight loss after new open gastric partitioning bypass procedure so that such improvements can lead to salvage of potentially lost years of life $[22,23]$.

To prevent weight gain in the future, a common issue in restrictive methods, our technique combines malabsorptive and restrictive processes. Although currently available procedures such as LRYGB offers combined malabsorptive and restrictive processes as well, GPB has several advantages in comparison with LRYGB. In LRYGB the pouch is completely detached from remaining part of stomach which would make EGD very challenging; however, the anatomic structure of the stomach is preserved in GPB which would not interfere with EGD. The second advantage of GPB is lower incidence of nutritional deficiencies secondary to malabsorption. Traditional LRYGB completely bypasses the absorptive segments of small intestine leading to nutritional 
deficiencies such as anemia, and osteoporosis [24-26]. In the GPB technique, however, gastric contents are divided in two routes: the Roux limb (which receives the majority of gastric outflow) and the intestine (through Pylorus and thereby allowing early mixing with biliary and pancreatic enzymes resulting in sufficient absorption of nutrients). This advantage of GPB results in passage of larger portion of gastric contents through the Roux limb and smaller portion through the more physiologic small intestine, which in turn would prevent nutritional deficiencies. The latter mechanism comes at the price of slightly less weight loss such that prior studies have demonstrated \%EWL of up to 75\% in LRYGB $[5,14,18,27]$ whereas \%EWL in GPB was $60 \%$. This degree of weight loss is still more prominent compared to restrictive techniques such as LAGB (41 - 47 \% EWL in four years) $[14,18,19,23,28,29]$.

This study is not without limitations. We performed this study as a proof of principle and examined the effectiveness and side effect profile of this novel technique in a small sample size of 30 patients. Larger studies with longer follow-up periods are warranted to further evaluate the persistence of weight loss and side effects. Secondly, we performed the procedure with laparotomy, which would make it less favorable for surgeons and patients. We are currently developing the laparoscopic technique of the same procedure, which is expected to have similar efficacy, less side effects, and decreased hospital stay.

In conclusion, the new open gastric partitioning bypass procedure utilizes both malabsorptive and restrictive techniques in bariatric surgery, which in first study on 30 patients showed promising results. Due to its exclusive characteristics, the weight loss effect lies between LAGB and LRYGB methods, but in contrast to those procedures, it is cheaper, is easier to perform, and has less side effects. Subsequent larger studies are warranted to further evaluate the long term effects.

\section{Ethical Approval:}

All procedures performed in this study were in accordance with the ethical standards of the institutional and national research committee and with the 1964 Helsinki declaration and its later amendments or comparable ethical standards.

\section{References}

1. Ogden CL, Carroll MD, Kit BK, Flegal KM (2014) Prevalence of childhood and adult obesity in the United States, 2011-2012. JAMA 311: 806-14.

2. Esteghamati A, Khalilzadeh O, Mohammad K, Meysamie A, Rashidi A, et al. (2010) Secular trends of obesity in Iran between 1999 and 2007: National Surveys of Risk Factors of Non-communicable Diseases. Metab Syndr Relat Disord 8: 209-13.

3. Hosseinpanah F, Barzin M, Amiri P, Azizi F (2011) The trends of metabolic syndrome in normal-weight Tehranian adults. Ann Nutr Metab 58: 126-32.

4. Pi-Sunyer FX (1999) Comorbidities of overweight and obesity: current evidence and research issues. Med Sci Sports Exerc 31: S602-8.

5. McGraw CA, Wool DB (2015) Bariatric surgery: three surgical techniques, patient care, risks, and outcomes. AORN journal 102: 141-52.

6. Colquitt JL, Picot J, Loveman E, Clegg AJ (2009) Surgery for obesity. Cochrane Database Syst Rev 2: 10.1002/14651858.CD003641

7. Eliakim A, Friedland O, Kowen G, Wolach B, Nemet D (2004) Parental obesity and higher pre-intervention BMI reduce the likelihood of a multidisciplinary childhood obesity program to succeed-a clinical observation. J Pediatr Endocrinol Metab 17: 1055-61.

8. Buchwald H, Williams SE (2004) Bariatric Surgery Worldwide 2003. Obes Surg 14: 1157-64.

9. Eckhout GV, Willbanks OL, Moore JT (1986) Vertical ring gastroplasty for morbid obesity: five year experience with 1,463 patients. Am J Surg 152: 713-6.

10. Mason EE (1982) Vertical banded gastroplasty for obesity. Arch Surg 117: 701-6.

11. Saber AA, Elgamal MH, McLeod MK (2008) Bariatric surgery: the past, present, and future. Obes Surg 18: 121-8.

12. Victorzon M, Tolonen P (2013) Mean fourteen-year, 100\% follow-up of laparoscopic adjustable gastric banding for morbid obesity. Surg Obes Relat Dis 9 : 753-7.

13. Awruch D, Escalona A, Gamboa C, Salinas J, Ramírez SR, et al. (2009) PL-122: Laparoscopic Roux-en-Y gastric bypass (LRYGB) versus laparoscopic adjustable gastric banding (LAGB) 5 years follow-up. Surg Obes Relat Dis 5: S8.

14. Boza C, Gamboa C, Awruch D, Perez G, Escalona A, et al. (2010) Laparoscopic Roux-en-Y gastric bypass versus laparoscopic adjustable gastric banding: five years of follow-up. Surg Obes Relat Dis 6: 470-5.

15. Shen X, Zhang X, Bi J, Yin K (2014) Long-term complications requiring reoperations after laparoscopic adjustable gastric banding: a systematic review. Surg Obes Relat Dis 11: 956-64.

16. Liu XZ, Yin K, Fan J, Shen XJ, Xu MJ, et al. (2014) Long-Term outcomes and experience of laparoscopic adjustable gastric banding: one center's results in China. Surg Obes Relat Dis 11: 855-9

17. Sarela AI, Dexter SP, O’Kane M, Menon A, McMahon MJ (2012) Long-term follow-up after laparoscopic sleeve gastrectomy: 8-9-year results. Surg Obes Relat Dis 8: 679-84.

18. Angrisani L, Cutolo PP, Formisano G, Nosso G, Vitolo G (2013) Laparoscopic adjustable gastric banding versus Roux-en-Y gastric bypass: 10-year results of a prospective, randomized trial. Surg Obes Relat Dis 9: 405-13.

19. Vu L, Switzer NJ, De Gara C, Karmali S (2013) Surgical interventions for obesity and metabolic disease. Best Best Pract Res Clin Endocrinol Metab 27: 239-46.

20. DeMaria EJ (2007) Bariatric surgery for morbid obesity. N Engl J Med 356: 2176-83.

21. Korenkov M, Sauerland S (2007) Clinical update: bariatric surgery. Lancet 370: 1988-90.

22. Fontaine KR, Redden DT, Wang C, Westfall AO, Allison DB (2003) Years of life lost due to obesity. JAMA 289: 187-93.

23. Angrisani L, Lorenzo M, Borrelli V (2007) Laparoscopic adjustable gastric banding versus Roux-en-Y gastric bypass: 5-year results of a prospective randomized trial. Surg Obes Relat Dis 3: 127-32.

24. Goldner WS, O’Dorisio TM, Dillon JS, Mason EE (2002) Severe metabolic bone disease as a long-term complication of obesity surgery. Obes Surg 12: 685-92.

25. Halverson JD, Zuckerman GR, Koehler RE, Gentry K, Michael H, et al. (1981) Gastric bypass for morbid obesity: a medical--surgical assessment. Ann Surg 194: 152-60. 
26. Van Osdol A, Borgert A, Kallies K, Kothari S, Grover B (2015) A5082-Micronutrient Deficiencies In Patients After Laparoscopic Roux-En-Y Gastric Bypass Versus Sleeve Gastrectomy. Surg Obes Relat Dis 11: S98.

27. Boza C, Gamboa C, Salinas J, Achurra P, Vega A, et al. (2012) Laparoscopic Roux-en-Y gastric bypass versus laparoscopic sleeve gastrectomy: a case-control study and 3 years of follow-up. Surg Obes Relat Dis 8: 243-9.

28. Franco JV, Ruiz PA, Palermo M, Gagner M (2011) A review of studies comparing three laparoscopic procedures in bariatric surgery: sleeve gastrectomy, Rouxen-Y gastric bypass and adjustable gastric banding. Obes Surg 21: 1458-68.

29. Himpens J, Dapri G, Cadière GB (2006) A prospective randomized study between laparoscopic gastric banding and laparoscopic isolated sleeve gastrectomy: results after 1 and 3 years. Obes Surg 16: 1450-6. 